\title{
Rayleigh-Quotient Representation of the Real Parts, Imaginary Parts, and Moduli of the Eigenvalues of Diagonalizable Matrices
}

\author{
Ludwig Kohaupt ${ }^{1}$ \\ ${ }^{1}$ Department of Mathematics, Beuth University of Technology Berlin, Berlin, Germany
}

\section{Article Info}

Keywords: Asymptotic stability of dynamical systems, Circular damped eigenfrequencies, Moduli of eigenvalues, Rayleigh quotient, Real and imaginary parts of eigenvalues, Weighted norm.

2010 AMS: 11E39, 15A18, 15B57,

15B99, 65F35, 65J05.

Received: 28 November 2018

Accepted: 17 January 2019

Available online: 30 August 2019

\begin{abstract}
In the present paper, formulas for the Rayleigh-quotient representation of the real parts, imaginary parts, and moduli of the eigenvalues of diagonalizable matrices are obtained that resemble corresponding formulas for the eigenvalues of self-adjoint matrices. These formulas are new and of interest in Linear Algebra and in the theory of linear dynamical systems. Since the style of paper is expository, it could also be of interest in graduate/undergraduate teaching or research at college level. The key point is that a weighted scalar product is used that is defined by means of a special positive definite matrix. As applications, one obtains convexity properties of newly-defined numerical ranges of a matrix. A numerical example underpins the theoretical findings.
\end{abstract}

\section{Introduction}

For self-adjoint matrices, there are formulas for the eigenvalues in the form of Rayleigh quotients; more precisely, max-, min-, minmax-, and maxmin-formulas are known; for this, see, e.g., the book [12, Section 5.4].

In the present paper, we obtain corresponding formulas for the real and imaginary parts as well as for the moduli of the eigenvalues of a diagonalizable matrix. First, these formulas are of interest on their own in Linear Algebra. Second, these are also of potential interest, for example, in the theory of linear dynamical systems. The reason for this is as follows. The real parts of the eigenvalues multiplied by the time are equal to the arguments of the exponential functions that describe the decay behavior of the solution (see, e.g., [4, Section 7.1, p.2011, Formulas (89), (90)]). Further, the system is asymptotically stable if the real parts of all eigenvalues are negative. Moreover, when the eigenvalues are pairwise conjugate-complex, then the moduli of the imaginary parts are the circular damped eigenfrequencies of the system (see, e.g., [4, Section 7.1, p. 2011, (89)]).

The paper is structured as follows.

In Section 2, preliminary materials are assembled on biorthogonality relations for the eigenvectors of a diagonalizable matrix $A$ and the eigenvectors of $A^{*}$ that will be useful in the sequel. Moreover, the construction of positive semi-definite matrices $R_{j}$ and of the positive definite matrix $R=\sum_{j=1}^{n} R_{j}$ is reviewed where the last one is employed to define a weighted scalar product $(\cdot, \cdot)_{R}$ that plays a key role in deriving the new results. In Sections 3, 4, and 5, formulas for the Rayleigh-quotient representation of the real parts, imaginary parts, and moduli of the eigenvalues of a diagonalizable matrix are given, as the case may be. In Section 6, a connection between the matrices $R^{-1} \frac{A^{*} R+R A}{2}, R^{-1} \frac{R A-A^{*} R}{2 i}$, and $R^{-1} A^{*} R A$ is established that play a key role in the study of the real parts, imaginary parts, and moduli of the eigenvalues of $A$, respectively. Section 7 describes the applications and Section 8 a numerical example. Finally, Section 9 contains the conclusions and an outlook on to future work. The non-cited references [1], [7], [8], [9], [10], and [11] are given because they may be useful to the reader in the context of the present paper.

\section{Preliminaries}

As a preparation to Theorem 2.1, we formulate the following conditions $(C 1)-(C 4)$ : 
(C1) $A \in C^{n \times n}$

(C2) A is diagonalizable, and $\lambda_{i}, i=1, \cdots, n$ are the eigenvalues of $A$ as well as $p_{i}, i=1, \cdots, n$ the associated eigenvectors

(C3) $u_{i}^{*}, i=1, \cdots, n$ are the eigenvectors of $A^{*}$ corresponding to the eigenvalues $\overline{\lambda_{i}}, i=1, \cdots, n$ of $A^{*}$

(C4) $\lambda_{i} \neq \lambda_{j}, i \neq j, i, j=1, \cdots, n$

Based on Theorem 2.1, in Theorem 2.2 the positive semi-definite matrices $R_{j}$ and the positive definite matrix $R=\sum_{j=1}^{n} R_{j}$ are constructed; they play a key role in the subsequent sections.

We mention that, even though condition $(C 4)$ may be omitted, it is nevertheless useful here since it will turn out to be fulfilled in the numerical example in Section 8 and since the biorthogonal system in Theorem 2.1 can be constructed more easily than without this condition. As a consequence, also the matrices $R_{j}$ and $R$ can be computed more easily than without condition $(C 4)$.

The material in this section is known, i.e. this is a review section. Therefore, the compiled results are given without proofs. But, it is indicated where the proofs can be found. Now, two theorems follow.

Theorem 2.1. (Biorthogonality relations with $\lambda_{i} \neq \lambda_{j}, i \neq j, i, j=1, \cdots, n$ )

Let the conditions $(C 1)-(C 4)$ be fulfilled. Then, after appropriate normalization of the eigenvectors $p_{i}, i=1, \cdots, n$ and $u_{i}^{*}, i=1, \cdots, n$, one has the biorthogonality relations

$$
\left(p_{i}, u_{j}^{*}\right)=\delta_{i j}, i, j=1, \cdots, n
$$

where $(\cdot, \cdot)$ is the usual scalar product on $C^{n}$.

Proof. See [2, Theorem 1].

Theorem 2.2. (Construction of positive definite matrix $R$ )

Let the conditions $(C 1)$ - (C4) be fulfilled. Let $\alpha_{j}=\lambda_{j}(A)$ be the eigenvalues and $u_{j}$ be the associated left eigenvectors of $A$ for $j=1, \cdots, n$; further, let $A^{*} \in C^{n \times n}$ be the adjoint matrix of $A$ so that $u_{j}^{*}$ are the right eigenvectors of $A^{*}$ corresponding to the eigenvalues $\overline{\alpha_{j}}$ of $A^{*}$ for $j=1, \cdots, n$, i.e.,

$$
u_{j} A=\alpha_{j} u_{j}, j=1, \cdots, n
$$

and

$$
A^{*} u_{j}^{*}=\overline{\alpha_{j}} u_{j}^{*}, j=1, \cdots, n .
$$

Let

$$
\begin{gathered}
\rho_{j}=\overline{\alpha_{j}}+\alpha_{j}=2 \operatorname{Re} \alpha_{j}=2 \operatorname{Re} \overline{\alpha_{j}}, j=1, \cdots, n, \\
\sigma_{j}=\alpha_{j}-\overline{\alpha_{j}}=2 i \operatorname{Im} \alpha_{j}, j=1, \cdots, n,
\end{gathered}
$$

and

$$
R_{j}=u_{j}^{*} u_{j}, j=1, \cdots, n
$$

Then,

$$
\begin{aligned}
& A^{*} R_{j}+R_{j} A=\rho_{j} R_{j}, j=1, \cdots, n, \\
& R_{j} A-A^{*} R_{j}=\sigma_{j} R_{j}, j=1, \cdots, n .
\end{aligned}
$$

In other words: The matrix eigenvalue problem

$$
A^{*} V+V A=\mu V
$$

has the $\mathrm{n}$ solution pairs

$$
(\mu, V)=\left(\rho_{j}, R_{j}\right)
$$

with real $\rho_{j}$, and the matrix eigenvalue problem

$$
V A-A^{*} V=\mu V
$$

has the $\mathrm{n}$ solution pairs

$$
(\mu, V)=\left(\sigma_{j}, R_{j}\right)
$$

with purely imaginary $\sigma_{j}$.

The matrices $R_{j} \in C^{n \times n}$ are positive semi-definite for $j=1, \cdots, n$. Further,

$$
R:=\sum_{j=1}^{n} R_{j}
$$

is positive definite. 
Proof. The assertions on (2.4) and on $R_{j}$ and $R$ follow from [5, Theorems 4 - 6]. The proof of (2.5) is derived in a similar way as for (2.4).

Remark 2.3. Since $R$ in (2.6) is positive definite, by

$$
(u, v)_{R}:=(R u, v), u, v \in C^{n},
$$

$a$ weighted scalar product $(\cdot, \cdot)_{R}$ is defined and by

$$
\|u\|_{R}:=(R u, u)^{\frac{1}{2}}, u \in C^{n}
$$

$a$ weighted norm $\|\cdot\|_{R}$.

Remark 2.4. For definiteness, one can assume condition (C4) for Theorem 2.2. But, as [3, Theorem 4] shows, Theorem 2.2 is valid without this condition.

\section{Formulas for the representation of the real parts of the eigenvalues of a diagonalizable matrix}

In this section, we want to derive formulas for the representation of the real parts of the eigenvalues of a diagonalizable matrix $A$ by Rayleigh quotients. More precisely, max-, min-, minmax-, and maxmin-representations are obtained corresponding to associated formulas for the eigenvalues of self-adjoint matrices, assembled, for instance, in the book [12, Section 5.4].

First, we want to derive a relation corresponding to that of [12, Section 5.4(18)]. This is done in the following Formula (3.2).

Lemma 3.1. Let the conditions $(C 1)$ - $(C 4)$ be fulfilled. Then, with the denotations of Theorem 2.2 ,

$$
(A u, u)_{R}=\sum_{j=1}^{n} \lambda_{j}(A)(u, u)_{R_{j}}, u \in C^{n}
$$

leading to

$$
\operatorname{Re}(A u, u)_{R}=\sum_{j=1}^{n} \operatorname{Re} \lambda_{j}(A)(u, u)_{R_{j}}, u \in C^{n} .
$$

If matrix $A$ is, beyond this, asymptotically stable, i.e., if

$$
\operatorname{Re} \lambda_{j}(A)<0, j=1, \cdots, n,
$$

then

$$
\operatorname{Re}(A u, u)_{R}=-\sum_{j=1}^{n}\left|\operatorname{Re} \lambda_{j}(A)\right|(u, u)_{R_{j}}, u \in C^{n}
$$

so that, in this case,

$$
\operatorname{Re}(A u, u)_{R}<0,0 \neq u \in C^{n}
$$

and

$$
\left|\operatorname{Re}(A u, u)_{R}\right|=\sum_{j=1}^{n}\left|\operatorname{Re} \lambda_{j}(A)\right|(u, u)_{R_{j}}, u \in C^{n} .
$$

Proof. Let $u \in C^{n}$. Then from Theorem 2.1,

$$
u=\sum_{j=1}^{n}\left(u, u_{j}^{*}\right) p_{j}
$$

implying

$$
\begin{aligned}
(A u, u)_{R} & =(R A u, u)=(A u, R u) \\
& =\sum_{j, k=1}^{n}\left(u, u_{j}^{*}\right) \overline{\left(u, u_{k}^{*}\right)}\left(A p_{j}, R p_{k}\right) \\
& =\sum_{j, k=1}^{n}\left(u, u_{j}^{*}\right) \overline{\left(u, u_{k}^{*}\right)} \lambda_{j}\left(p_{j}, R p_{k}\right) \\
& =\sum_{j, k=1}^{n}\left(u, u_{j}^{*}\right) \overline{\left(u, u_{k}^{*}\right)} \lambda_{j}\left(p_{j}, u_{k}^{*}\right) \\
& =\sum_{j=1}^{n} \lambda_{j}\left|\left(u, u_{j}^{*}\right)\right|^{2} \\
& =\sum_{j=1}^{n} \lambda_{j}(u, u)_{R_{j}}
\end{aligned}
$$


since

$$
(u, u)_{R_{j}}=\left(R_{j} u, u\right)=\left(u_{j}^{*} u_{j} u, u\right)=\left(u_{j} u, u_{j} u\right)=\left|u_{j} u\right|^{2}=\left|\left(u, u_{j}^{*}\right)\right|^{2} .
$$

So, (3.1) is proven. The rest is clear.

Next, we have

Lemma 3.2. Let the conditions $(C 1)$ - $(C 4)$ be fulfilled. Further, let matrix $A$ be asymptotically stable. Then, $A^{*} R+R A$ is negative definite.

Proof. From (2.4) and (2.2), we obtain

$$
\begin{aligned}
\left(-\left[A^{*} R+R A\right] u, u\right) & =\sum_{j=1}^{n}\left(-\rho_{j}\right)(u, u)_{R_{j}}=2 \sum_{j=1}^{n} \operatorname{Re}\left(-\lambda_{j}(A)\right)(u, u)_{R_{j}} \\
& =2 \sum_{j=1}^{n}\left|\operatorname{Re} \lambda_{j}(A)\right|(u, u)_{R_{j}} \\
& \geq 2 \min _{j=1, \cdots, n}\left|\operatorname{Re} \lambda_{j}(A)\right| \sum_{j=1}^{n}(u, u)_{R_{j}} \\
& =c_{0}(R u, u)>0,0 \neq u \in C^{n}
\end{aligned}
$$

with $c_{0}=2 \min _{j=1, \cdots, n}\left|\operatorname{Re} \lambda_{j}(A)\right|>0$.

Next, we want to define vector spaces similar to those in [12, Section 5.4,(23)].

Comparing the formulas in [12, Section 5.4,(17)] and (3.2), one is led to define here the following vector spaces.

$$
M_{1}:=C^{n}, M_{k}:=\left\{u \in C^{n} \mid(u, u)_{R_{i}}=0, i=1,2, \cdots, k-1\right\}, k=2, \cdots, n .
$$

The next lemma characterizes these spaces.

Lemma 3.3. Let the conditions $(C 1)$ - $(C 4)$ be fulfilled as well as $\left\{p_{1}, \cdots, p_{n}\right\}$ and $\left\{u_{1}^{*}, \cdots, u_{n}^{*}\right\}$ be a biorthogonal set of eigenvectors of $A$ and $A^{*}$, i.e., such that

$$
\left(p_{j}, u_{k}^{*}\right)=\delta_{j k}, j, k=1, \cdots, n
$$

Then,

$$
M_{k}=\left[p_{k}, p_{k+1}, \cdots, p_{n}\right], k=1, \cdots, n .
$$

Proof. The proof is done for $k=3$. The general case can be made by induction. So, we have to prove

$$
M_{3}=\left\{u \in C^{n} \mid(u, u)_{R_{1}}=0,(u, u)_{R_{2}}=0\right\}=\left[p_{3}, p_{4}, \cdots, p_{n}\right]
$$

(i) $\left[p_{3}, p_{4}, \cdots, p_{n}\right] \subset M_{3}$ :

Let $u \in\left[p_{3}, p_{4}, \cdots, p_{n}\right]$. Then, $u=\sum_{j=3}^{n} \beta_{j} p_{j}$ with elements $\beta_{j} \in C, j=3, \cdots, n$. Let $s \in\{1,2\}$. This entails, due to Theorem 2.1,

$$
\begin{aligned}
(u, u)_{R_{s}} & =\sum_{j, k=3}^{n} \beta_{j} \overline{\beta_{k}}\left(p_{j}, p_{k}\right)_{R_{s}}=\sum_{j, k=3}^{n} \beta_{j} \overline{\beta_{k}}\left(u_{s}^{*} u_{s} p_{j}, p_{k}\right) \\
& =\sum_{j, k=3}^{n} \beta_{j} \overline{\beta_{k}}\left(u_{s}^{*}\left(u_{s} p_{j}\right), p_{k}\right)=\sum_{j, k=3}^{n} \beta_{j} \overline{\beta_{k}}\left(u_{s}^{*}\left(p_{j}, u_{s}^{*}\right), p_{k}\right) \\
& =\sum_{j, k=3}^{n} \beta_{j} \overline{\beta_{k}} \delta_{s j}\left(u_{s}^{*}, p_{k}\right)=\sum_{j, k=3}^{n} \beta_{j} \overline{\beta_{k}} \delta_{s j} \delta_{s k}=0 .
\end{aligned}
$$

Therefore, $(u, u)_{R_{s}}=0, s=1,2$ and thus $u \in M_{3}$ so that $\left[p_{3}, p_{4}, \cdots, p_{n}\right] \subset M_{3}$ is proven.

(ii) $M_{3} \subset\left[p_{3}, p_{4}, \cdots, p_{n}\right]$ :

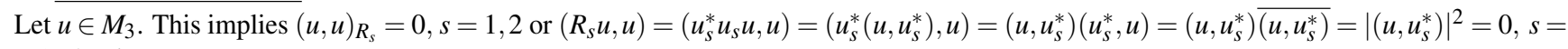
1,2 , that is,

$$
\left(u, u_{s}^{*}\right)=0, s=1,2
$$

Thus,

$$
u \in\left[u_{1}^{*}, u_{2}^{*}\right]^{\perp}
$$

Now,

$$
\left[u_{1}^{*}, u_{2}^{*}\right] \oplus\left[u_{1}^{*}, u_{2}^{*}\right]^{\perp}=C^{n} .
$$

Since also

$$
\left[u_{1}^{*}, u_{2}^{*}\right] \oplus\left[u_{3}^{*}, u_{4}^{*}, \cdots, u_{n}^{*}\right]=C^{n}
$$


we deduce

$$
\left[u_{1}^{*}, u_{2}^{*}\right]^{\perp}=\left[u_{3}^{*}, u_{4}^{*}, \cdots, u_{n}^{*}\right] .
$$

So, according to (3.14) and (3.15),

$$
u \in\left[u_{3}^{*}, u_{4}^{*}, \cdots, u_{n}^{*}\right]
$$

Thus, there exist elements $\gamma_{3}, \cdots, \gamma_{n} \in C$ such that

$$
u=\sum_{j=3}^{n} \gamma_{j} u_{j}^{*} \in\left[p_{1}, p_{2}\right]^{\perp}=\left[p_{3}, p_{4}, \cdots, p_{n}\right]
$$

This completes the proof of the assertion.

Similarly to [12, Section 5.4,(22)], we suppose that the eigenvalues $\lambda_{1}(A), \cdots, \lambda_{n}(A)$ of matrix $A$ are arranged such that

$$
\operatorname{Re} \lambda_{1}(A) \geq \operatorname{Re} \lambda_{2}(A) \geq \cdots \geq \operatorname{Re} \lambda_{n}(A)
$$

If $A$ is asymptotically stable, (3.16) is replaced by

$$
\left|\operatorname{Re} \lambda_{1}(A)\right| \geq\left|\operatorname{Re} \lambda_{2}(A)\right| \geq \cdots \geq\left|\operatorname{Re} \lambda_{n}(A)\right| .
$$

One has

Theorem 3.4. Let the conditions $(C 1)$ - $(C 4)$ be fulfilled. Further, let the eigenvalues of $A$ be arranged according to (3.16). Moreover, let the vector spaces $M_{k}, k=1, \cdots, n$ be defined by (3.11) or (3.12).

Then,

$$
\operatorname{Re} \lambda_{k}(A)=\max _{0 \neq u \in M_{k}} \frac{\operatorname{Re}(A u, u)_{R}}{(u, u)_{R}}, k=1,2, \cdots, n .
$$

If matrix $A$ is, beyond this, asymptotically stable, i.e., if (3.2) is valid and if the eigenvalues are arranged according to (3.17), then also

$$
\left|\operatorname{Re} \lambda_{k}(A)\right|=\max _{0 \neq u \in M_{k}} \frac{\left|\operatorname{Re}(A u, u)_{R}\right|}{(u, u)_{R}}, k=1,2, \cdots, n .
$$

The maximum is attained for $u=p_{k}$.

Proof. There are two methods to derive this theorem.

Method 1: One uses equation (3.2) as starting point, i.e.,

$$
\operatorname{Re}(A u, u)_{R}=\sum_{j=1}^{n} \operatorname{Re} \lambda_{j}(A)\left(R_{j} u, u\right), u \in C^{n} .
$$

Choosing $k \in\{1, \cdots, n\}$ fixed and $u \in M_{k}$, using (3.11), one obtains

$$
\begin{aligned}
\operatorname{Re}(A u, u)_{R} & =\sum_{j=k}^{n} \operatorname{Re} \lambda_{j}(A)\left(R_{j} u, u\right) \leq \max _{j=k, \cdots, n} \operatorname{Re} \lambda_{j}(A) \sum_{j=k}^{n}\left(R_{j} u, u\right) \\
& =\operatorname{Re} \lambda_{k}(A) \sum_{j=1}^{n}\left(R_{j} u, u\right)=\operatorname{Re} \lambda_{k}(A)(u, u)_{R},
\end{aligned}
$$

that is,

$$
\frac{\operatorname{Re}(A u, u)_{R}}{(u, u)_{R}} \leq \operatorname{Re} \lambda_{k}(A), 0 \neq u \in M_{k}
$$

and thus

$$
\max _{0 \neq u \in M_{k}} \frac{\operatorname{Re}(A u, u)_{R}}{(u, u)_{R}} \leq \operatorname{Re} \lambda_{k}(A) .
$$

Now, the maximum is attained for $u=p_{k} \in M_{k}$, that is,

$$
\operatorname{Re} \lambda_{k}(A)=\frac{\operatorname{Re}\left(A p_{k}, p_{k}\right)_{R}}{\left(p_{k}, p_{k}\right)_{R}} \leq \max _{0 \neq u \in M_{k}} \frac{\operatorname{Re}(A u, u)_{R}}{(u, u)_{R}} \leq \operatorname{Re} \lambda_{k}(A)
$$

so that the assertion (3.18) is proven.

Relation (3.19) is proven in the same way as (3.18), but based on (3.6) instead of (3.2) and (3.17) instead of (3.16).

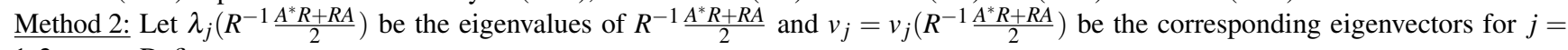
$1,2, \cdots, n$. Define

$$
M_{1}^{(R)}:=C^{n}, M_{k}^{(R)}:=\left\{u \in C^{n} \mid\left(u, v_{j}\right)_{R}=0, j=1, \cdots, k-1\right\}, k=2, \cdots, n
$$


From [12, Section 5.4(24)], it follows that

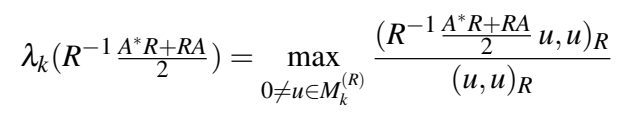

as $R^{-1} \frac{A^{*} R+R A}{2}$ is self-adjoint in the scalar product $(\cdot, \cdot)_{R}$. Since from $[6$, Theorem $6,(25)]$

$$
\lambda_{k}\left(R^{-1} \frac{A^{*} R+R A}{2}\right)=\operatorname{Re} \lambda_{k}(A)
$$

and further

$$
\left(R^{-1} \frac{A^{*} R+R A}{2} u, u\right)_{R}=\operatorname{Re}(A u, u)_{R}, u \in C^{n}
$$

we obtain

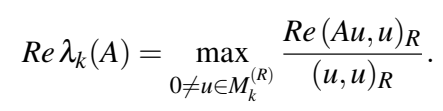

Now, also due to [6, Theorem 6, (26)],

$$
v_{j}=v_{j}\left(R^{-1} \frac{A^{*} R+R A}{2}\right)=p_{j}=p_{j}(A), j=1,2, \cdots, n .
$$

Therefore,

$$
\begin{aligned}
\left(u, v_{j}\right)_{R} & =\sum_{l=1}^{n}\left(u, R_{l} v_{j}\right)=\sum_{l=1}^{n}\left(u, u_{l}^{*} u_{l} p_{j}\right) \\
& =\sum_{l=1}^{n}\left(u, u_{l}^{*}\left(p_{j}, u_{l}^{*}\right)\right)=\sum_{l=1}^{n}\left(u, u_{l}^{*} \delta_{j l}\right) \\
& =\left(u, u_{j}^{*}\right), j=1,2, \cdots, k-1
\end{aligned}
$$

so that

$$
\begin{aligned}
M_{k}^{(R)} & =\left\{u \in C^{n} \mid\left(u, v_{j}\right)_{R}=0, j=1, \cdots, k-1\right\} \\
& =\left\{u \in C^{n} \mid\left(u, u_{j}^{*}\right)=0, j=1, \cdots, k-1\right\} \\
& =\left[p_{k}, p_{k+1}, \cdots, p_{n}\right]=M_{k}, k=1,2, \cdots, n .
\end{aligned}
$$

Thus, Formula (3.18) is proven.

Summarizing, in the first method, one follows a similar way as in the case of self-adjoint matrices $A$, and in the second method, one reduces the case of diagonalizable matrices to the case of self-adjoint matrices by using a self-adjoint matrix that has the eigenvalues $\operatorname{Re} \lambda_{k}(A)$.

From the two proofs of Theorem 3.4, it is clear that one can carry over many results from the self-adjoint case to the case of diagonalizable matrices. In the sequel, we shall do so essentially without giving proofs.

So, we have the following further Rayleigh-quotient representations corresponding to [12, Subsection 5.4, (25)] in the case of self-adjoint matrices.

One has the following theorem.

Theorem 3.5. Let the conditions $(C 1)$ - $(C 4)$ be fulfilled. Further, let the eigenvalues of $A$ be arranged according to (3.16). Then, for every $j=1, \cdots, n$ and every subspace $M \subset C^{n}$ with $\operatorname{dim} M=m=n+1-j$, the following inequalities are valid:

$$
\operatorname{Re} \lambda_{j}(A) \leq \max _{0 \neq u \in M} \frac{\operatorname{Re}(A u, u)_{R}}{(u, u)_{R}} \leq \operatorname{Re} \lambda_{1}(A)
$$

and the following representation formulas hold:

$$
\operatorname{Re} \lambda_{j}(A)=\min _{\operatorname{dim} M=m 0 \neq u \in M} \frac{\operatorname{Re}(A u, u)_{R}}{(u, u)_{R}} .
$$

If matrix $A$ is, beyond this, asymptotically stable and the eigenvalues are arranged according to (3.17), then also

$$
\left|\operatorname{Re} \lambda_{j}(A)\right|=\min _{\operatorname{dim} M=m 0 \neq u \in M} \frac{\left|\operatorname{Re}(A u, u)_{R}\right|}{(u, u)_{R}} .
$$

Remark 3.6. From (3.21), it follows

$$
\frac{\operatorname{Re}(A u, u)_{R}}{(u, u)_{R}} \leq v[A]=\max _{j=1, \cdots, n} \operatorname{Re} \lambda_{j}(A), 0 \neq u \in C^{n} .
$$

For the following theorem, we need the vector spaces $N_{k}$ defined by

$$
N_{k}:=\left[p_{1}, p_{2}, \cdots, p_{k}\right], k=1,2, \cdots, n .
$$

Then, we have a result similar to that of Theorem 3.4. 
Theorem 3.7. Let the conditions $(C 1)$ - $(C 4)$ be fulfilled. Further, let the eigenvalues of $A$ be arranged according to (3.16). Moreover, let the vector spaces $N_{k}, k=1, \cdots, n$ be defined by (3.24).

Then,

$$
\operatorname{Re} \lambda_{k}(A)=\min _{0 \neq u \in N_{k}} \frac{\operatorname{Re}(A u, u)_{R}}{(u, u)_{R}}, k=1,2, \cdots, n .
$$

If matrix $A$ is, beyond this, asymptotically stable, i.e., if (3.3) is valid and if the eigenvalues are arranged according to (3.17), then also

$$
\left|\operatorname{Re} \lambda_{k}(A)\right|=\min _{0 \neq u \in N_{k}} \frac{\left|\operatorname{Re}(A u, u)_{R}\right|}{(u, u)_{R}}, k=1,2, \cdots, n .
$$

The minimum is attained for $u=p_{k}$.

Next, we want to state a maxmin characterization for the real parts of eigenvalues similar to results for the eigenvalues in [12, Subsection 5.4, (26)].

One has the following theorem.

Theorem 3.8. Let the conditions $(C 1)$ - $(C 4)$ be fulfilled. Further, let the eigenvalues of $A$ be arranged according to (3.16). Then, for every $j=1, \cdots, n$ and every subspace $N \subset C^{n}$ with $\operatorname{dim} N=j$, the following inequalities are valid:

$$
\operatorname{Re} \lambda_{n}(A) \leq \min _{0 \neq u \in N} \frac{\operatorname{Re}(A u, u)_{R}}{(u, u)_{R}} \leq \operatorname{Re} \lambda_{j}(A),
$$

and the following representation formulas hold:

$$
\operatorname{Re} \lambda_{j}(A)=\max _{\operatorname{dim} N=j} \min _{0 \neq u \in N} \frac{\operatorname{Re}(A u, u)_{R}}{(u, u)_{R}} .
$$

If matrix $A$ is, beyond this, asymptotically stable and the eigenvalues are arranged according to (3.17), then also

$$
\left|\operatorname{Re} \lambda_{j}(A)\right|=\max _{\operatorname{dim} N=j} \min _{0 \neq u \in N} \frac{\left|\operatorname{Re}(A u, u)_{R}\right|}{(u, u)_{R}} .
$$

Remark 3.9. From (3.27), it follows

$$
\frac{\operatorname{Re}(A u, u)_{R}}{(u, u)_{R}} \geq-v[-A]=\min _{j=1, \cdots, n} \operatorname{Re} \lambda_{j}(A), 0 \neq u \in C^{n} .
$$

\section{Formulas for the representation of the imaginary parts of the eigenvalues of a diagonalizable matrix}

In this section, we want to state formulas for the representation of the imaginary parts of the eigenvalues of a diagonalizable matrix $A$ by Rayleigh quotients. More precisely, max-, min-, minmax-, and maxmin-representations are obtained corresponding to associated formulas for the eigenvalues of self-adjoint matrices, assembled, for instance, in the textbook [12, Section 5.4] resp. corresponding to those for the real parts of diagonalizable matrices in Section 3.

First, we want to state a relation corresponding to that of [12, Section 5.4(18)]. This is done in the following Formula (4.1).

Lemma 4.1. Let the conditions $(C 1)$ - $(C 4)$ be fulfilled. Then, with the denotations of Theorem 2.1,

$$
\operatorname{Im}(A u, u)_{R}=\sum_{j=1}^{n} \operatorname{Im} \lambda_{j}(A)(u, u)_{R_{j}}, u \in C^{n} .
$$

Proof. Equation (4.1) follows directly from Lemma 3.1, Formula (3.1).

Similarly to [12, Section 5.4,(22)] or (3.16), we suppose that the eigenvalues $\lambda_{1}(A), \cdots, \lambda_{n}(A)$ of matrix $A$ are arranged such that

$$
\operatorname{Im} \lambda_{1}(A) \geq \operatorname{Im} \lambda_{2}(A) \geq \cdots \geq \operatorname{Im} \lambda_{n}(A) .
$$

Then, one has a series of theorems for the imaginary parts of the eigenvalues corresponding to those of Theorems $3.4-3.8$.

Theorem 4.2. Let the conditions $(C 1)$ - $(C 4)$ be fulfilled. Further, let the eigenvalues of $A$ be arranged according to to (4.2). Moreover, let the vector spaces $M_{k}, k=1, \cdots, n$ be defined by (3.11) or (3.12).

Then,

$$
\operatorname{Im} \lambda_{k}(A)=\max _{0 \neq u \in M_{k}} \frac{\operatorname{Im}(A u, u)_{R}}{(u, u)_{R}}, k=1,2, \cdots, n .
$$

The maximum is attained for $u=p_{k}$.

Proof. As for Theorem 3.4, there are two methods to derive this theorem.

Method 1: One uses equation (4.1) as starting point and proceeds as in Method 1 for the proof of Theorem 3.4.

Method 2: Let $\lambda_{j}\left(R^{-1} \frac{R A-A^{*} R}{2 i}\right)$ be the eigenvalues of $R^{-1} \frac{R A-A^{*} R}{2 i}$ and $v_{j}=v_{j}\left(R^{-1} \frac{R A-A^{*} R}{2 i}\right)$ be the corresponding eigenvectors for $j=1, \cdots, n$. Then, with [6, Theorem 6], the assertion follows in a similar way as in Method 2 for the proof of Theorem 3.4. 
Next, we state a minmax characterization for the imaginary parts of eigenvalues similar to results for the eigenvalues in [12, Section 5.4, (25)] or for the real parts of the eigenvalues in Theorem 3.5.

One has the following theorem.

Theorem 4.3. Let the conditions $(C 1)$ - $(C 4)$ be fulfilled. Further, let the eigenvalues of $A$ be arranged according to (4.2). Then, for every $j=1, \cdots, n$ and every subspace $M \subset C^{n}$ with $\operatorname{dim} M=m=n+1-j$, the following inequalities are valid:

$$
\operatorname{Im} \lambda_{j}(A) \leq \max _{0 \neq u \in M} \frac{\operatorname{Im}(A u, u)_{R}}{(u, u)_{R}} \leq \operatorname{Im} \lambda_{1}(A)
$$

and the following representation formulas hold:

$$
\operatorname{Im} \lambda_{j}(A)=\min _{\operatorname{dim} M=m 0 \neq u \in M} \max _{0} \frac{\operatorname{Im}(A u, u)_{R}}{(u, u)_{R}} .
$$

Remark 4.4. From (4.4), it follows

$$
\frac{\operatorname{Im}(A u, u)_{R}}{(u, u)_{R}} \leq \max _{j=1, \cdots, n} \operatorname{Im} \lambda_{j}(A), 0 \neq u \in C^{n}
$$

Next, we have a result similar to that of Theorem 4.2.

Theorem 4.5. Let the conditions $(C 1)$ - $(C 4)$ be fulfilled. Further, let the eigenvalues of $A$ be arranged according to (4.2). Moreover, let the vector spaces $N_{k}, k=1, \cdots, n$ be defined by (3.24).

Then,

$$
\operatorname{Im} \lambda_{k}(A)=\min _{0 \neq u \in N_{k}} \frac{\operatorname{Im}(A u, u)_{R}}{(u, u)_{R}}, k=1,2, \cdots, n
$$

The minimum is attained for $u=p_{k}$.

Next, we state a maxmin characterization for the imaginary parts of eigenvalues similar to results for the eigenvalues in [12, Subsection 5.4, (26)] for self-adjoint matrices or for the real parts in Theorem 3.8.

One has the following theorem.

Theorem 4.6. Let the conditions $(C 1)$ - $(C 4)$ be fulfilled. Further, let the eigenvalues of $A$ be arranged according to (4.2). Then, for every $j=1, \cdots, n$ and every subspace $N \subset C^{n}$ with $\operatorname{dim} N=j$, the following inequalities are valid:

$$
\operatorname{Im} \lambda_{n}(A) \leq \min _{0 \neq u \in N} \frac{\operatorname{Im}(A u, u)_{R}}{(u, u)_{R}} \leq \operatorname{Im} \lambda_{j}(A)
$$

and the following representation formulas hold:

$$
\operatorname{Im} \lambda_{j}(A)=\max _{\operatorname{dim} N=j} \min _{0 \neq u \in N} \frac{\operatorname{Im}(A u, u)_{R}}{(u, u)_{R}} .
$$

\section{Formulas for the representation of the moduli of the eigenvalues of a diagonalizable matrix}

In this section, we want to state formulas for the representation of the moduli of the eigenvalues of a diagonalizable matrix $A$ by Rayleigh quotients. More precisely, max-, min-, minmax-, and maxmin-representations are obtained corresponding to associated formulas for the eigenvalues of self-adjoint matrices, assembled, for instance, in the textbook [12, Section 5.4] resp. corresponding to those for the real parts in Section 3 and for the imaginary parts in Section 4.

First, we want to give a relation corresponding to that of [12, Section 5.4(18)]. This is done in the following Formula (5.1).

Lemma 5.1. Let the conditions $(C 1)$ - $(C 4)$ be fulfilled. Then, with the denotations of Theorem 2.2,

$$
\|A u\|_{R}^{2}=\sum_{j=1}^{n}\left|\lambda_{j}(A)\right|^{2}\|u\|_{R_{j}}^{2}, u \in C^{n} .
$$

Proof. Let $u \in C^{n}$. From (3.7),

$$
u=\sum_{k=1}^{n}\left(u, u_{k}^{*}\right) p_{k}
$$

and thus,

$$
A u=\sum_{k=1}^{n}\left(u, u_{k}^{*}\right) \lambda_{k} p_{k}
$$

This entails

$$
\|A u\|_{R}^{2}=(R A u, A u)=\sum_{j=1}^{n}(A u, A u)_{R_{j}}=\sum_{j, k, i=1}^{n}\left(u, u_{k}^{*}\right) \lambda_{k} \overline{\left(u, u_{i}^{*}\right) \lambda_{i}}\left(p_{k}, p_{i}\right)_{R_{j}}
$$


Now, due to (2.1),

$$
\begin{aligned}
\left(p_{k}, p_{i}\right)_{R_{j}} & =\left(u_{j}^{*} u_{j} p_{k}, p_{i}\right)=\left(u_{j}^{*}\left(p_{k}, u_{j}^{*}\right), p_{i}\right) \\
& =\left(p_{k}, u_{j}^{*}\right)\left(u_{j}^{*}, p_{i}\right)=\left(p_{k}, u_{j}^{*}\right) \overline{\left(p_{i}, u_{j}^{*}\right)}=\delta_{k j} \delta_{i j}
\end{aligned}
$$

Therefore, from (5.4) and (5.5),

$$
\|A u\|_{R}^{2}=\sum_{k=1}^{n}\left|\lambda_{k}\right|^{2}\left|\left(u, u_{k}^{*}\right)\right|^{2}
$$

Taking into account (3.9), leads to the assertion.

Similarly to [12, Section 5.4,(22)] or (3.16) resp. (4.2), we suppose in this section that the moduli of the eigenvalues $\lambda_{1}(A), \cdots, \lambda_{n}(A)$ of matrix $A$ are arranged such that

$$
\left|\lambda_{1}(A)\right| \geq\left|\lambda_{2}(A)\right| \geq \cdots \geq\left|\lambda_{n}(A)\right| .
$$

Then, one has a series of theorems for the moduli of the eigenvalues corresponding to Theorems $3.4-3.8$ and Theorems 4.2 -4.6.

Theorem 5.2. Let the conditions $(C 1)$ - $(C 4)$ be fulfilled. Further, let the moduli of the eigenvalues of $A$ be arranged according to (5.6). Moreover, let the vector spaces $M_{k}, k=1, \cdots, n$ be defined by (3.11) or (3.12).

Then,

$$
\left|\lambda_{k}(A)\right|=\max _{0 \neq u \in M_{k}} \frac{\|A u\|_{R}}{\|u\|_{R}}, k=1,2, \cdots, n .
$$

Proof. As in Theorems 3.4 and 4.2, there are two methods to derive this theorem.

Method 1: According to (5.1), one has

$$
\|A u\|_{R}^{2}=\sum_{j=1}^{n}\left|\lambda_{j}(A)\right|^{2}\|u\|_{R_{j}}^{2}, u \in C^{n} .
$$

Choosing $k \in\{1, \cdots, n\}$ fixed and $u \in M_{k}$, one obtains

$$
\begin{aligned}
\|A u\|_{R}^{2} & =\sum_{j=k}^{n}\left|\lambda_{j}(A)\right|^{2}\|u\|_{R_{j}}^{2} \leq \max _{j=k, \cdots, n}\left|\lambda_{j}(A)\right|^{2} \sum_{j=k}^{n}\|u\|_{R_{j}}^{2} \\
& =\left|\lambda_{k}(A)\right|^{2} \sum_{j=1}^{n}\|u\|_{R_{j}}^{2}=\left|\lambda_{k}(A)\right|^{2}\|u\|_{R}^{2},
\end{aligned}
$$

that is,

$$
\frac{\|A u\|_{R}^{2}}{\|u\|_{R}^{2}} \leq\left|\lambda_{k}(A)\right|^{2}, 0 \neq u \in M_{k}
$$

and thus

$$
\max _{0 \neq u \in M_{k}} \frac{\|A u\|_{R}}{\|u\|_{R}} \leq\left|\lambda_{k}(A)\right| .
$$

Now, the maximum is attained for $u=p_{k} \in M_{k}$, that is,

$$
\left|\lambda_{k}(A)\right|=\frac{\left\|A p_{k}\right\|_{R}}{\left\|p_{k}\right\|_{R}} \leq \max _{0 \neq u \in M_{k}} \frac{\|A u\|_{R}}{\|u\|_{R}} \leq\left|\lambda_{k}(A)\right| .
$$

so that the assertion (5.7) is proven.

Method 2:

Let $\lambda_{j}\left(R^{-1} A^{*} R A\right)$ be the eigenvalues of $R^{-1} A^{*} R A$ and $v_{j}=v_{j}\left(R^{-1} A^{*} R A\right)$ be the corresponding eigenvectors for $j=1,2, \cdots, n$. With the spaces $M_{k}^{(R)}$ defined in the proof of Theorem 3.4, from [12, Section 5.4(24)], it follows that

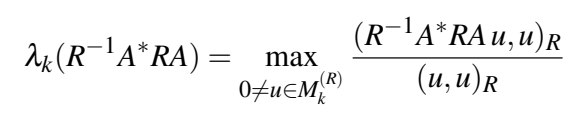

as $R^{-1} A^{*} R A$ is self-adjoint in the scalar product $(\cdot, \cdot)_{R}$. Since from [6, Theorem 7, (29)],

$$
\lambda_{k}\left(R^{-1} A^{*} R A\right)=\left|\lambda_{k}(A)\right|^{2}
$$

and further

$$
\left(R^{-1} A^{*} R A u, u\right)_{R}=(A u, A u)_{R}=\|A u\|_{R}^{2}, u \in C^{n},
$$


we obtain

$$
\left|\lambda_{k}(A)\right|^{2}=\max _{0 \neq u \in M_{k}^{(R)}} \frac{\|A u\|_{R}^{2}}{\|u\|_{R}^{2}}
$$

and thus

$$
\left|\lambda_{k}(A)\right|=\max _{0 \neq u \in M_{k}^{(R)}} \frac{\|A u\|_{R}}{\|u\|_{R}} .
$$

Now, also due to [6, Theorem 7,(30)],

$$
v_{j}=v_{j}\left(R^{-1} A^{*} R A\right)=p_{j}=p_{j}(A), j=1,2, \cdots, n .
$$

Therefore, as in Section 3,

$$
\left(u, v_{j}\right)_{R}=\left(u, u_{j}^{*}\right), j=1,2, \cdots, k-1
$$

and

$$
M_{k}^{(R)}=\left[p_{k}, p_{k+1}, \cdots, p_{n}\right]=M_{k}, k=1,2, \cdots, n .
$$

Thus, Formula (5.7) is proven.

Next, we want to state a minmax characterization for the moduli of eigenvalues similar to results for the eigenvalues in [12, Subsection 5.4, (25)].

One has the following theorem whose proof is conducted along the lines of Method 1 or Method 2 in the proof of Theorem 5.2.

Theorem 5.3. Let the conditions $(C 1)$ - $(C 4)$ be fulfilled. Further, let the eigenvalues of $A$ be arranged according to (5.6). Then, for every $j=1, \cdots, n$ and every subspace $M \subset C^{n}$ with $\operatorname{dim} M=m=n+1-j$, the following inequalities are valid:

$$
\left|\lambda_{j}(A)\right| \leq \max _{0 \neq u \in M} \frac{\|A u\|_{R}}{\|u\|_{R}} \leq\left|\lambda_{1}(A)\right|
$$

and the following representation formulas hold:

$$
\left|\lambda_{j}(A)\right|=\min _{\operatorname{dim} M=m=n+1-j 0 \neq u \in M} \frac{\|A u\|_{R}}{\|u\|_{R}} .
$$

Remark 5.4. From (5.9), it follows

$$
\frac{\|A u\|_{R}}{\|u\|_{R}} \leq \rho(A)=\max _{j=1, \cdots, n}\left|\lambda_{j}(A)\right|, 0 \neq u \in C^{n}
$$

The next theorems correspond to Theorems 3.7 and 3.8 resp. Theorems 4.5 and 4.6.

Theorem 5.5. Let the conditions $(C 1)$ - $(C 4)$ be fulfilled. Further, let the moduli of the eigenvalues of $A$ be arranged according to (5.6). Moreover, let the vector spaces $N_{k}, k=1, \cdots, n$ be defined by (3.24).

Then,

$$
\left|\lambda_{k}(A)\right|=\min _{0 \neq u \in N_{k}} \frac{\|A u\|_{R}}{\|u\|_{R}}, k=1,2, \cdots, n .
$$

Finally, one has the following theorem.

Theorem 5.6. Let the conditions $(C 1)$ - $(C 4)$ be fulfilled. Further, let the moduli of the eigenvalues of $A$ be arranged according to (5.6). Then, for every $j=1, \cdots, n$ and every subspace $N \subset C^{n}$ with $\operatorname{dim} N=j$, the following inequalities are valid:

$$
\left|\lambda_{n}(A)\right| \leq \min _{0 \neq u \in N} \frac{\|A u\|_{R}}{\|u\|_{R}} \leq\left|\lambda_{j}(A)\right|
$$

and the following representation formulas hold:

$$
\left|\lambda_{j}(A)\right|=\max _{\operatorname{dim} N=j} \min _{0 \neq u \in N} \frac{\|A u\|_{R}}{\|u\|_{R}} .
$$

Remark 5.7. From (5.12), it follows

$$
\frac{\|A u\|_{R}}{\|u\|_{R}} \geq \min _{j=1, \cdots, n}\left|\lambda_{j}(A)\right|=\left[\rho\left(A^{-1}\right)\right]^{-1}, 0 \neq u \in C^{n}
$$

where the last equal sign holds if $A$ is nonsingular. If $A$ is singular, then $\left[\rho\left(A^{-1}\right)\right]^{-1}$ has to be interpreted as zero. 


\section{Connection between the matrices $R^{-1} \frac{A^{*} R+R A}{2}, R^{-1} \frac{R A-A^{*} R}{2 i}$, and $R^{-1} A^{*} R A$}

The equation

$$
(\operatorname{Re} z)^{2}+(\operatorname{Im} z)^{2}=|z|^{2}
$$

is valid for all $z \in C$.

Now, let $A \in C^{n \times n}, n \in N, j \in\{1, \ldots, n\}$, and $\lambda_{j}(A)$ be an eigenvalue of $A$. Set $z=\lambda_{j}(A)$. Then,

$$
\left|\lambda_{j}(A)\right|^{2}=\left(\operatorname{Re} \lambda_{j}(A)\right)^{2}+\left(\operatorname{Im} \lambda_{j}(A)\right)^{2} .
$$

According to [6, Theorems 6 and 7],

$$
\begin{aligned}
& \operatorname{Re} \lambda_{j}(A)=\lambda_{j}\left(R^{-1} \frac{A^{*} R+R A}{2}\right), \\
& \operatorname{Im} \lambda_{j}(A)=\lambda_{j}\left(R^{-1} \frac{R A-A^{*} R}{2 i}\right),
\end{aligned}
$$

and

$$
\left|\lambda_{j}(A)\right|^{2}=\lambda_{j}\left(R^{-1} A^{*} R A\right)
$$

leading to

$$
\left(\lambda_{j}\left(R^{-1} \frac{A^{*} R+R A}{2}\right)\right)^{2}+\left(\lambda_{j}\left(R^{-1} \frac{R A-A^{*} R}{2 i}\right)\right)^{2}=\lambda_{j}\left(R^{-1} A^{*} R A\right)
$$

or

$$
\lambda_{j}\left(\left(R^{-1} \frac{A^{*} R+R A}{2}\right)^{2}\right)+\lambda_{j}\left(\left(R^{-1} \frac{R A-A^{*} R}{2 i}\right)^{2}\right)=\lambda_{j}\left(R^{-1} A^{*} R A\right)
$$

From this relation, the question arises as to whether a similar relation also holds for the pertinent matrices. This turns out to be indeed true. Namely, we have

Theorem 6.1. Let the conditions $(C 1)-(C 4)$ be fulfilled. Then,

$$
\left(R^{-1} \frac{A^{*} R+R A}{2}\right)^{2}+\left(R^{-1} \frac{R A-A^{*} R}{2 i}\right)^{2}=R^{-1} A^{*} R A .
$$

Proof. The proof is based on [6, Theorems 6 and 7]. One has

$$
\begin{aligned}
& {\left[\left(R^{-1} \frac{A^{*} R+R A}{2}\right)^{2}+\left(R^{-1} \frac{R A-A^{*} R}{2 i}\right)^{2}\right] p_{j} } \\
= & \left(R^{-1} \frac{A^{*} R+R A}{2}\right)^{2} p_{j}+\left(R^{-1} \frac{R A-A^{*} R}{2 i}\right)^{2} p_{j} \\
= & {\left[\operatorname{Re} \lambda_{j}(A)\right]^{2} p_{j}+\left[\operatorname{Im} \lambda_{j}(A)\right]^{2} p_{j} } \\
= & \left|\lambda_{j}(A)\right|^{2} p_{j}=\lambda_{j}\left(R^{-1} A^{*} R A\right) p_{j}=R^{-1} A^{*} R A p_{j},
\end{aligned}
$$

$j=1, \cdots, n$ so that

$$
\left[\left(R^{-1} \frac{A^{*} R+R A}{2}\right)^{2}+\left(R^{-1} \frac{R A-A^{*} R}{2 i}\right)^{2}\right] u=R^{-1} A^{*} R A u, u \in C^{n}
$$

which is equivalent to (6.7).

\section{Applications}

In this section, we apply the results of Sections 3, 4, and 5 to obtain the convexity of newly-defined numerical ranges of a diagonalizable matrix $A$.

\subsection{Applications pertinent to Section 3}

In this subsection, we first generalize the notion of the numerical range of a matrix $A$ with respect to the ordinary scalar product $(\cdot, \cdot)$ on $C^{n}$, denoted by $W_{(\cdot,)}(A)$, to the corresponding notion with weighted scalar product $W_{(\cdot, \cdot)_{R}}(A)$.

As the next step, the real part of the numerical range $W_{(\cdot,)_{R}}(A)$ will be defined, denoted by $\operatorname{Re}\left[W_{(\cdot, \cdot)_{R}}(A)\right]$.

Further, the real part of the spectrum $\operatorname{Re}[\sigma(A)]$ is introduced.

Based on these notions, some applications of the derived results are obtained.

For example, the alternative form

$$
\operatorname{Re}\left[W_{(\cdot,)_{R}}(A)\right]=W_{(\cdot, \cdot)_{R}}\left(R^{-1} \frac{A^{*} R+R A}{2}\right)
$$

is derived where the matrix $R^{-1} \frac{A^{*} R+R A}{2}$ is regular if $A$ is asymptotically stable. 
Let the conditions $(C 1)$ - $(C 4)$ be fulfilled and the matrices $R_{j}$ and $R$ be given by (2.3) and (2.6).

The numerical range of $A$ with respect to the scalar product $(\cdot, \cdot)$ is defined by

$$
W_{(\cdot, \cdot)}(A)=\left\{z \in C \mid z=\frac{(A u, u)}{(u, u)}, 0 \neq u \in C^{n}\right\},
$$

cf. [12, Section 5.4]. Accordingly, we define

$$
W_{(\cdot, \cdot)_{R}}(A)=\left\{z \in C \mid z=\frac{(A u, u)_{R}}{(u, u)_{R}}, 0 \neq u \in C^{n}\right\} .
$$

Further, let

$$
\operatorname{Re}\left[W_{(\cdot,)_{R}}(A)\right]:=\left\{x \in R \mid x=\frac{\operatorname{Re}(A u, u)_{R}}{(u, u)_{R}}, 0 \neq u \in C^{n}\right\} ;
$$

we call it real part of the numerical range $W_{(\cdot, \cdot)_{R}}(A)$.

Let $\sigma(A)=\left\{\lambda_{j}(A), j=1, \cdots, n\right\}$ be the spectrum of $A$, i.e., the set of all eigenvalues of $A$.

Similarly as before, we define

$$
\operatorname{Re}[\sigma(A)]:=\left\{\operatorname{Re} \lambda_{j}(A), j=1, \cdots, n\right\}
$$

and call it the real part of the spectrum of $A$.

Finally, let $c o\{\operatorname{Re}[\sigma(A)]\}$ be the convex hull of $\operatorname{Re}[\sigma(A)]$.

Next, we show the following corollary as an application of Theorem 3.5, Formula (3.21), and Theorem 3.8, Formula (3.27).

Corollary 7.1. (Application 1)

Let the conditions $(C 1)-(C 4)$ be fulfilled. Then, the set $\operatorname{Re}\left[W_{(\cdot, \cdot)_{R}}(A)\right]$ is convex, and one has the chain of equations

$$
\begin{aligned}
\operatorname{Re}\left[W_{(\cdot,)_{R}}(A)\right] & =\left\{x \in R \mid x=\frac{\operatorname{Re}(A u, u)_{R}}{(u, u)_{R}}, 0 \neq u \in C^{n}\right\} \\
& =\left\{x \in R \mid x=\frac{\left(R^{-1} \frac{A^{*} R+R A}{2} u, u\right)_{R}}{(u, u)_{R}}, 0 \neq u \in C^{n}\right\} \\
& =W_{(\cdot,)_{R}}\left(R^{-1} \frac{A^{*} R+R A}{2}\right) \\
& =\operatorname{co}\{\operatorname{Re}[\sigma(A)]\} .
\end{aligned}
$$

If the eigenvalues of $A$ are arranged according to (3.16), then

$$
\operatorname{Re}\left[W_{(\cdot, \cdot)_{R}}(A)\right]=\left[\operatorname{Re} \lambda_{n}(A), \operatorname{Re} \lambda_{1}(A)\right] .
$$

Proof. Let $0 \neq u \in C^{n}$. Then,

$$
2 \frac{\operatorname{Re}(A u, u)_{R}}{(u, u)_{R}}=\frac{\left(\left[A^{*} R+R A\right] u, u\right)}{(u, u)_{R}}=\frac{\left(R^{-1}\left[A^{*} R+R A\right] u, u\right)_{R}}{(u, u)_{R}} .
$$

The convexity follows from the last form with $R^{-1}\left[A^{*} R+R A\right]$ and the scalar product $(\cdot, \cdot)_{R}$, see the convexity of the numerical range of a matrix due to Hausdorff in [12, Section 5.4]. Since, with (3.16), one has

$$
\operatorname{co}\{\operatorname{Re}[\sigma(A)]\}=\left[\operatorname{Re} \lambda_{n}(A), \operatorname{Re} \lambda_{1}(A)\right],
$$

it remains to show that

$$
\operatorname{Re}\left[W_{(\cdot,)_{R}}(A)\right]=\left[\operatorname{Re} \lambda_{n}(A), \operatorname{Re} \lambda_{1}(A)\right]
$$

The proof of this relation is as follows.

(i) $\operatorname{Re}\left[W_{(\cdot,)_{R}}(A)\right] \subset\left[\operatorname{Re} \lambda_{n}(A), \operatorname{Re} \lambda_{1}(A)\right]$

This inclusion can be deduced from (3.21) with $\operatorname{dim} M=m=n-j+1$ for $j=1$ and (3.27) with $\operatorname{dim} N=n$. Namely, from (3.21), for $j=1$ and $\operatorname{dim} M=n$, i.e., $M=C^{n}$, one has

$$
\max _{0 \neq u \in C^{n}} \frac{\operatorname{Re}(A u, u)_{R}}{(u, u)_{R}} \leq \operatorname{Re} \lambda_{1}(A)
$$

and from (3.27), for $j=n$ and $\operatorname{dim} N=n$, i.e., $N=C^{n}$,

$$
\min _{0 \neq u \in C^{n}} \frac{\operatorname{Re}(A u, u)_{R}}{(u, u)_{R}} \geq \operatorname{Re} \lambda_{n}(A)
$$

(ii) $\left[\operatorname{Re} \lambda_{n}(A), \operatorname{Re} \lambda_{1}(A)\right] \subset \operatorname{Re}\left[W_{(\cdot, \cdot)_{R}}(A)\right]$

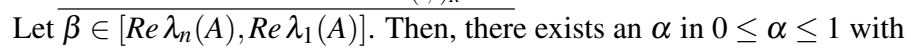

$$
\beta=\alpha \operatorname{Re} \lambda_{n}(A)+(1-\alpha) \operatorname{Re} \lambda_{1}(A) .
$$


Now, with the eigenvectors $p_{n}$ and $p_{1}$,

$$
\operatorname{Re} \lambda_{n}(A)=\frac{\operatorname{Re}\left(A p_{n}, p_{n}\right)_{R}}{\left(p_{n}, p_{n}\right)_{R}} \in \operatorname{Re}\left[W_{(\cdot, \cdot)_{R}}(A)\right]
$$

and

$$
\operatorname{Re} \lambda_{1}(A)=\frac{\operatorname{Re}\left(A p_{1}, p_{1}\right)_{R}}{\left(p_{1}, p_{1}\right)_{R}} \in \operatorname{Re}\left[W_{(\cdot,)_{R}}(A)\right] .
$$

Thus, due to the convexity of $\operatorname{Re}\left[W_{(\cdot,)_{R}}(A)\right]$, it follows that $\beta \in \operatorname{Re}\left[W_{(\cdot, \cdot)_{R}}(A)\right]$.

Corollary 7.2. (Application 2)

Let the conditions $(C 1)$ - $(C 4)$ be fulfilled. Further, let $A$ be asymptotically stable. Then,

$$
\operatorname{Re}\left[W_{(\cdot,)_{R}}(A)\right] \subset \boldsymbol{R}^{-}=\{x \in R \mid x<0\} .
$$

If $A$ is only stable, then

$$
\operatorname{Re}\left[W_{(\cdot, \cdot)_{R}}(A)\right] \subset \boldsymbol{R}_{0}^{-}=\{x \in R \mid x \leq 0\} .
$$

Proof. The first assertion follows from (3.5). The second assertion follows in a similar way.

\subsection{Applications pertinent to Section 4}

In this section, we proceed in a similar way as in 7.1. So, let

$$
\operatorname{Im}\left[W_{(\cdot, \cdot)_{R}}(A)\right]=\left\{x \in R \mid x=\frac{\operatorname{Im}(A u, u)_{R}}{(u, u)_{R}}, 0 \neq u \in C^{n}\right\} ;
$$

we call it the imaginary part of the numerical range $W_{(\cdot,)_{R}}(A)$.

Further, we define

$$
\operatorname{Im}[\sigma(A)]:=\left\{\operatorname{Im} \lambda_{j}(A), j=1, \cdots, n\right\}
$$

and call it the imaginary part of the spectrum of $A$.

Finally, let $\operatorname{co}\{\operatorname{Im}[\sigma(A)]\}$ be the convex hull of $\operatorname{Im}[\sigma(A)]$.

Herewith, we obtain

Corollary 7.3. (Application 3 )

Let the conditions $(C 1)-(C 4)$ be fulfilled. Then, the set $\operatorname{Im}\left[W_{(\cdot,)_{R}}(A)\right]$ is convex, and one has the chain of equations

$$
\begin{aligned}
\operatorname{Im}\left[W_{(\cdot, \cdot)_{R}}(A)\right] & =\left\{x \in R \mid x=\frac{\operatorname{Im}(A u, u)_{R}}{(u, u)_{R}}, 0 \neq u \in C^{n}\right\} \\
& =\left\{x \in R \mid x=\frac{\left(R^{-1} \frac{R A-A^{*} R}{2 i} u, u\right)_{R}}{(u, u)_{R}}, 0 \neq u \in C^{n} \cdot\right\} \\
& =W_{(\cdot,)_{R}}\left(R^{-1} \frac{R A-A^{*} R}{2 i}\right) \\
& =\operatorname{co}\{\operatorname{Im}[\sigma(A)]\} .
\end{aligned}
$$

If the eigenvalues of $A$ are arranged according to (4.2), then

$$
\operatorname{Im}\left[W_{(\cdot, \cdot)_{R}}(A)\right]=\left[\operatorname{Im} \lambda_{n}(A), \operatorname{Im} \lambda_{1}(A)\right] .
$$

Proof. The proof is similar to that of Corollary 7.1 and therefore omitted.

\subsection{Applications pertinent to Section 5}

In this subsection, we continue along the same lines as in 7.1 and 7.2.

Thus, let

$$
W_{\|\cdot\|_{R}}(A):=\left\{x \in R_{0}^{+} \mid x=\frac{\|A u\|_{R}}{\|u\|_{R}}, 0 \neq u \in C^{n}\right\} ;
$$

and call it the numerical range of $A$ with respect to the norm $\|\cdot\|_{R}$.

Further, we define

$$
|\sigma(A)|:=\left\{\left|\lambda_{j}(A)\right|, j=1, \cdots, n\right\}
$$

and call it the modulus of the spectrum of $A$.

Moreover, let $\operatorname{co}\{|\sigma(A)|\}$ be the convex hull of $|\sigma(A)|$.

Finally, let $S \subset R_{0}^{+}$be any set. We define

$$
S^{2}:=\left\{y \mid y=s^{2}, s \in S\right\}
$$

Next, we show the following corollary. 
Corollary 7.4. (Application 4)

Let the conditions $(C 1)-(C 4)$ be fulfilled. Then, the set $\left[W_{\|\cdot\|_{R}}(A)\right]^{2}$ is convex, and one has the chain of equations

$$
\begin{aligned}
{\left[W_{\|\cdot\|_{R}}(A)\right]^{2} } & =\left\{x \in R_{0}^{+} \mid x=\left[\frac{\|A u\|_{R}}{\|u\|_{R}}\right]^{2}=\frac{\|A u\|_{R}^{2}}{\|u\|_{R}^{2}}, 0 \neq u \in C^{n}\right\} \\
& =\left\{x \in R_{0}^{+} \mid x=\frac{\left(\left[R^{-1} A^{*} R A\right] u, u\right)_{R}}{(u, u)_{R}}, 0 \neq u \in C^{n}\right\} \\
& =W_{(\cdot, \cdot)_{R}}\left(R^{-1} A^{*} R A\right) \\
& =\operatorname{co}\left\{|\sigma(A)|^{2}\right\},
\end{aligned}
$$

and $R^{-1} A^{*} R A$ is self-adjoint and positive semi-definite in the weighted scalar product $(\cdot, \cdot)_{R}$. If $A$ is regular, then $R^{-1} A^{*} R A$ is apparently positive definite.

Further, if the moduli of the eigenvalues of $A$ are arranged according to (5.6), then

$$
\left[W_{\|\cdot\|_{R}}(A)\right]^{2}=\left[\left|\lambda_{n}(A)\right|^{2},\left|\lambda_{1}(A)\right|^{2}\right] .
$$

Proof. The proof is done by using Theorem 5.3, Formula (5.9) and Theorem 5.5, Formula (5.12).

Next, for $S \subset R_{0}^{+}$, we define

$$
\sqrt{S}:=\{y \mid y=\sqrt{s}, s \in S\} .
$$

Herewith, one can rewrite Corollary 7.4 in the following form.

Corollary 7.5. (Application 5)

Let the conditions $(C 1)$ - $(C 4)$ be fulfilled. Then, the set $W_{\|\cdot\|_{R}}(A)$ is convex, and one has the chain of equations

$$
\begin{aligned}
W_{\|\cdot\|_{R}}(A) & =\left\{x \in R_{0}^{+} \mid x=\frac{\|A u\|_{R}}{\|u\|_{R}}, 0 \neq u \in C^{n}\right\} \\
& =\left\{x \in R_{0}^{+} \mid x=\sqrt{\frac{\left(\left[R^{-1} A^{*} R A\right] u, u\right)_{R}}{(u, u)_{R}}}, 0 \neq u \in C^{n} \cdot\right\} \\
& =\sqrt{W_{(\cdot,)_{R}}\left(R^{-1} A^{*} R A\right)} \\
& =\sqrt{\operatorname{co}\left\{|\sigma(A)|^{2}\right\}} .
\end{aligned}
$$

If the moduli of the eigenvalues of $A$ are arranged according to (5.6), then

$$
W_{\|\cdot\|_{R}}(A)=\left[\left|\lambda_{n}(A)\right|,\left|\lambda_{1}(A)\right|\right] .
$$

Proof. For any subset $S \subset R_{0}^{+}$, one has

$$
\sqrt{S^{2}}=(\sqrt{S})^{2}=S
$$

Thus, from Corollary 7.4, the equations of Corollary 7.5 follow. Since, for the arrangement (5.6), $W_{\|\cdot\|_{R}}(A)=\left[\left|\lambda_{n}(A)\right|,\left|\lambda_{1}(A)\right|\right]$ and since the interval $\left[\left|\lambda_{n}(A)\right|,\left|\lambda_{1}(A)\right|\right]$ is convex, so is $W_{\|\cdot\|_{R}}(A)$.

\section{Numerical example}

In this section, we check the results of Subsection 7.1 as well as of Theorem 6.1, Formula (6.7) numerically. The numerical check of the results of Subsections 7.2 and 7.3 is left to the reader.

\subsection{A multi-mass vibration model}

We take up the multi-mass vibration model of [2], shown in Fig.1.

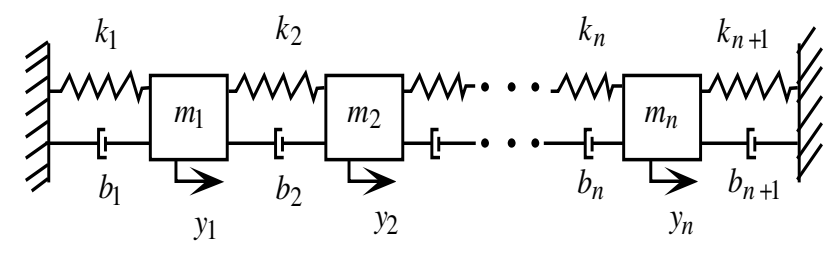


Fig.1: Multi-mass vibration model

The associated initial value problem is given by

$$
M \ddot{y}+B \dot{y}+K y=0, y(0)=y_{0}, \dot{y}(0)=\dot{y}_{0},
$$

where $y=\left[y_{1}, \cdots, y_{n}\right]^{T}$ and

$$
\begin{aligned}
& M=\left[\begin{array}{ccccc}
m_{1} & & & & \\
& m_{2} & & & \\
& & m_{3} & & \\
& & & \ddots & \\
& & & & m_{n}
\end{array}\right]
\end{aligned}
$$

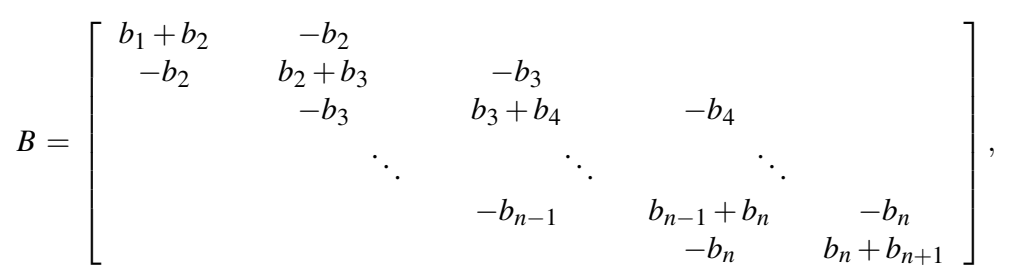

$$
\begin{aligned}
& K=\left[\begin{array}{cccccc}
k_{1}+k_{2} & -k_{2} & & & & \\
-k_{2} & k_{2}+k_{3} & -k_{3} & & & \\
& -k_{3} & k_{3}+k_{4} & -k_{4} & \\
& & \ddots & \ddots & \ddots & \\
& & & -k_{n-1} & k_{n-1}+k_{n} & -k_{n} \\
& & & & -k_{n} & k_{n}+k_{n+1}
\end{array}\right]
\end{aligned}
$$

with the mass, damping, and stiffness matrices $M, B$, and $K$, as the case may be, and the displacement vector $y$ as in [2]. In state-space description, this problem takes the form

$$
\dot{x}=A x, t \geq 0, x(0)=x_{0},
$$

where $x=\left[y^{T}, z^{T}\right]^{T}, z=\dot{y}$, and where the system matrix $A$ is given by

$$
A=\left[\begin{array}{c|c}
0 & E \\
\hline-M^{-1} K & -M^{-1} B
\end{array}\right] .
$$

\subsection{Data}

The values $m_{j}, j=1, \cdots, n$ and $b_{j}, k_{j}, j=1, \cdots, n+1$ are also specified as in [2], namely as

$$
\begin{aligned}
& m_{j}=1, j=1, \cdots, n \\
& \text { and } \\
& k_{j}=1, j=1, \cdots, n+1 \\
& b_{j}=\left\{\begin{array}{l}
1 / 2, j \text { even } \\
1 / 4, j \text { odd }
\end{array}\right.
\end{aligned}
$$

Then,

$$
B=\left[\begin{array}{rrrrr}
\frac{3}{4} & -\frac{1}{2} & & & \\
-\frac{1}{2} & \frac{3}{4} & -\frac{1}{4} & & \\
& -\frac{1}{4} & \frac{3}{4} & -\frac{1}{2} & \\
& & \ddots & \ddots & \ddots \\
& & -\frac{1}{4} & \frac{3}{4} & -\frac{1}{2} \\
& & & -\frac{1}{2} & \frac{3}{4}
\end{array}\right]
$$

(if $n$ is even), and

$$
K=\left[\begin{array}{rrrrr}
2 & -1 & & & \\
-1 & 2 & -1 & & \\
& -1 & 2 & -1 & \\
& & \ddots & \ddots & \ddots \\
& & -1 & 2 & -1 \\
& & & -1 & 2
\end{array}\right]
$$


We add the details from [2] in order to make the paper more readable on its own. Further, we choose $n=5$ in this paper so that the state-space vector has dimension $m=2 n=10$. For the initial time, we take

$$
t_{0}=0 .
$$

Finally, the initial conditions for $y(t)$ and $\dot{y}(t)$ can be chosen as

$$
y_{0}=[-1,1,-1,1,-1]^{T}
$$

as well as

$$
\dot{y}_{0}=[-1,-1,-1,-1,-1]^{T}
$$

(which corresponds to IC(II) in [2, p.159]). But, they are not needed here.

\subsection{Auxiliary computational results}

As in [2], using the Matlab routine eig.m, one obtains

$$
\begin{aligned}
& \lambda_{1}(A)=-0.050239361219464+0.516371450711010 i, \\
& \lambda_{2}(A)=-0.050239361219464-0.516371450711010 i . \\
& \lambda_{3}(A)=-0.187331625959257+0.994521686465592 i, \\
& \lambda_{4}(A)=-0.187331625959257-0.994521686465592 i, \\
& \lambda_{5}(A)=-0.375000000000000+1.363589014329465 i, \\
& \lambda_{6}(A)=-0.375000000000000-1.363589014329465 i, \\
& \lambda_{7}(A)=-0.562668374040742+1.616358701643860 i, \\
& \lambda_{8}(A)=-0.562668374040742-1.616358701643860 i, \\
& \lambda_{9}(A)=-0.699760638780536+1.795981478159753 i, \\
& \lambda_{10}(A)=-0.699760638780536-1.795981478159753 i .
\end{aligned}
$$

Therefore, $\lambda_{j}(A), j=1, \cdots, m=2 n=10$ are distinct. Thus, matrix $A$ is diagonalizable, regular, and asymptotically stable. The eigenvalues are ordered such that $\operatorname{Re} \lambda_{1}(A) \geq \cdots \geq \operatorname{Re} \lambda_{10}(A)$.

The weighted matrix $R$ is computed as

$R=\left[\begin{array}{rrrrrrrrrr}1.2501 & -0.2868 & -0.1297 & 0.0135 & -0.0988 & 0.1966 & -0.1314 & -0.3356 & -0.0196 & 0.2662 \\ -0.2868 & 1.0887 & -0.3824 & -0.0842 & 0.0179 & -0.1567 & 0.2066 & 0.0440 & 0.0239 & 0.0319 \\ -0.1297 & -0.3824 & 1.1899 & -0.3531 & -0.1196 & 0.2780 & 0.0018 & 0.2200 & -0.1271 & -0.3402 \\ 0.0135 & -0.0842 & -0.3531 & 1.0873 & -0.3227 & -0.0173 & -0.0525 & -0.1892 & 0.1947 & 0.0505 \\ -0.0988 & 0.0179 & -0.1196 & -0.3227 & 1.2619 & -0.3017 & 0.0042 & 0.3092 & 0.0215 & 0.2699 \\ 0.1966 & -0.1567 & 0.2780 & -0.0173 & -0.3017 & 0.7620 & 0.2782 & 0.1039 & 0.0439 & -0.0256 \\ -0.1314 & 0.2066 & 0.0018 & -0.0525 & 0.0042 & 0.2782 & 0.8373 & 0.3358 & 0.1512 & 0.0458 \\ -0.3356 & 0.0440 & 0.2200 & -0.1892 & 0.3092 & 0.1039 & 0.3358 & 0.9171 & 0.3240 & 0.1085 \\ -0.0196 & 0.0239 & -0.1271 & 0.1947 & 0.0215 & 0.0439 & 0.1512 & 0.3240 & 0.8373 & 0.2919 \\ 0.2662 & 0.0319 & -0.3402 & 0.0505 & 0.2699 & -0.0256 & 0.0458 & 0.1085 & 0.2919 & 0.7685\end{array}\right]$

\subsection{Numerical check of the validity of Corollary 21 (Application 1)}

Here, we check the validity of

$$
\frac{\operatorname{Re}(A u, u)_{R}}{(u, u)_{R}} \in \operatorname{Re}\left[W_{(\cdot, \cdot)_{R}}(A)\right]=\left[\operatorname{Re} \lambda_{10}(A), \operatorname{Re} \lambda_{1}(A)\right], 0 \neq u \in C^{10}
$$

and choose $u \in\left\{e, e_{1}, e_{2}, e_{1,2}\right\}$ where

$$
\begin{aligned}
& e=[1,1,1,1,1,1,1,1,1,1]^{T}, \\
& e_{1}=[1,0,0,0,0,0,0,0,0,0]^{T}, \\
& e_{2}=[0,1,0,0,0,0,0,0,0,0]^{T}, \\
& e_{1,2}=[1,1,0,0,0,0,0,0,0,0]^{T} .
\end{aligned}
$$

We obtain

$$
\left[\operatorname{Re} \lambda_{10}(A), \operatorname{Re} \lambda_{1}(A)\right]=[-0.699760638780535,-0.050239361219464]
$$

and further

$$
\begin{aligned}
& \frac{\operatorname{Re}(A e, e)_{R}}{(e, e)_{R}}=-0.076392659033957 \in\left[\operatorname{Re} \lambda_{10}(A), \operatorname{Re} \lambda_{1}(A)\right], \\
& \frac{\operatorname{Re}\left(A e_{1}, e_{1}\right)_{R}}{\left(e_{1}, e_{1}\right)_{R}}=-0.419568056678684 \in\left[\operatorname{Re} \lambda_{10}(A), \operatorname{Re} \lambda_{1}(A)\right], \\
& \frac{\operatorname{Re}\left(A e_{2}, e_{2}\right)_{R}}{\left(e_{2}, e_{2}\right)_{R}}=-0.483140758290161 \in\left[\operatorname{Re} \lambda_{10}(A), \operatorname{Re} \lambda_{1}(A)\right], \\
& \frac{\operatorname{Re}\left(A e_{1,2}, e_{1,2}\right)_{R}}{\left(e_{1,2}, e_{1,2}\right)_{R}}=-0.230440140020361 \in\left[\operatorname{Re} \lambda_{10}(A), \operatorname{Re} \lambda_{1}(A)\right] .
\end{aligned}
$$




\subsection{Numerical check of the validity of Theorem 6.1}

Here, we obtain

$\left(R^{-1} \frac{A^{*} R+R A}{2}\right)^{2}+\left(R^{-1} \frac{R A-A^{*} R}{2 i}\right)^{2} \doteq R^{-1} A^{*} R A \doteq$
$\left[\begin{array}{rrrrrrrrrr}2.0451 & -1.0850 & 0.0397 & -0.0038 & 0.0066 & -0.0013 & -0.1139 & -0.0054 & -0.0091 & 0.0044 \\ -1.0900 & 1.9991 & -0.9022 & -0.0478 & -0.0070 & -0.1139 & 0.0060 & 0.1256 & -0.0031 & -0.0078 \\ -0.0561 & -0.9022 & 2.0073 & -1.0978 & 0.0429 & -0.0054 & 0.1256 & 0.0104 & -0.1216 & -0.0090 \\ -0.0038 & 0.0480 & -1.0970 & 2.0007 & -0.8984 & -0.0091 & -0.0031 & -0.1216 & 0.0060 & 0.1347 \\ 0.0082 & -0.0078 & -0.0529 & -0.8931 & 1.9473 & 0.0044 & -0.0078 & -0.0090 & 0.1347 & 0.0029 \\ -0.1112 & 0.2210 & -0.1122 & 0.0172 & -0.0179 & 1.9892 & -1.0016 & 0.0107 & 0.0015 & 0.0010 \\ 0.2338 & -0.0003 & -0.2482 & 0.1239 & 0.0124 & -1.0016 & 1.9690 & -0.9964 & 0.0154 & -0.0020 \\ 0.1364 & -0.2461 & -0.0169 & 0.2447 & -0.1037 & 0.0107 & -0.9964 & 1.9701 & -1.0036 & 0.0192 \\ 0.0152 & -0.1246 & 0.2462 & 0.0010 & -0.2633 & 0.0015 & 0.0154 & -1.0036 & 1.9690 & -0.9979 \\ -0.0166 & 0.0110 & 0.1448 & -0.2754 & 0.1288 & 0.0010 & -0.0020 & 0.0192 & -0.9979 & 1.9788\end{array}\right]$

and, for

$$
D:=\left(R^{-1} \frac{A^{*} R+R A}{2}\right)^{2}+\left(R^{-1} \frac{R A-A^{*} R}{2 i}\right)^{2}-R^{-1} A^{*} R A,
$$

we get

$$
|D|_{\infty}:=\max _{j, k=1, \ldots, 10}\left|D_{j k}\right| \doteq 3.0002 \times 10^{-14} .
$$

So, Theorem 6.1 is numerically confirmed.

\subsection{Computational aspects}

In this subsection, we say something about the used computer equipment and the computation times.

(i) As to the computer equipment, the following hardware was available: an Intel Core2 Duo Processor at $3166 \mathrm{GHz}$, a $500 \mathrm{~GB}$ mass storage facility, and two $2048 \mathrm{MB}$ high-speed memories. As software package for the computations, we used MATLAB, Version 7.11.

(ii) The computation time $t$ of an operation was determined by the command sequence $t 1=$ clock; operation; $t=e t i m e($ clock, 1 ). It is put out in seconds, rounded to four decimal places. For the computation of the eigenvalues of matrix $A$ in Subsection 5.3, we used the command $[\mathrm{XA}, \mathrm{DA}]=\mathrm{eig}(\mathrm{A})$; the pertinent computation time was less than $0.0001 \mathrm{~s}$.

\section{Conclusion and outlook on to future work}

It has been shown that there exist Rayleigh-quotient representations of the real parts, imaginary parts, and moduli of the eigenvalues of diagonalizable matrices that parallel those representations known for the eigenvalues of self-adjoint matrices. The key idea is to use a weighted scalar product defined by a positive definite matrix that is constructed by means of left eigenvectors of the considered matrix and the right eigenvectors of its adjoint. The results are of interest on their own in Linear Algebra. They are also of potential interest in applications. For example, in the theory of linear dynamical systems, in the study of stability of a vibration problem, the real parts of the eigenvalues of the system matrix are important. Moreover, in systems with conjugate-complex eigenvalues, the moduli of the imaginary parts of the eigenvalues are the circular damped eigenfrequencies of the system. A somewhat surprising point is also the derived relation $\left(R^{-1} \frac{A^{*} R+R A}{2}\right)^{2}+\left(R^{-1} \frac{R A-A^{*} R}{2 i}\right)^{2}=R^{-1} A^{*} R A$. On the whole, the results should be of interest to mathematicians as well as engineers.

The case of general matrices is more involved and will be dealt with in a subsequent paper.

\section{References}

[1] A. Czornik, P. Jurgaś, Some properties of the spectral radius of a set of matrices, Int. J. Appl. Math. Sci. 16(2)(2006)183-188.

[2] L. Kohaupt, Construction of a biorthogonal system of principal vectors of the matrices $A$ and $A^{*}$ with applications to the initial value problem $\dot{x}=A x, x\left(t_{0}\right)=x_{0}$, J. Comp. Math. Opt. 3(3)(2007)163-192.

[3] L. Kohaupt, Biorthogonalization of the principal vectors for the matrices A and $A^{*}$ with application to the computation of the explicit representation of the solution $x(t)$ of $\dot{x}=A x, x\left(t_{0}\right)=x_{0}$, Appl. MAth. Sci. 2(20)(2008)961-974.

[4] L. Kohaupt, Solution of the vibration problem $M \ddot{y}+B \dot{y}+K y=0, y\left(t_{0}\right)=y_{0}, \dot{y}\left(t_{0}\right)=\dot{y}_{0}$ without the hypothesis $B M^{-1} K=K M^{-1} B$ or $B=\alpha M+\beta K$, Appl. MAth. Sci. 2(41)(2008)1989-2024.

[5] L. Kohaupt, Solution of the matrix eigenvalue problem $V A+A^{*} V=\mu V$ with applications to the study of free linear systems, J. Comp. Appl. Math. 213(1)(2008)142-165.

[6] L. Kohaupt, Spectral properties of the matrix $C^{-1} B$ with positive definite matrix $C$ and Hermitian B as well as applications, J. Appl. Math. Comput., DOI 10.1007/s12190-015-0876-8, (2015) 28 pages.

[7] T.J. Laffey, H. S̆migoc, Nonnegatively realizable spectra with two positive eigenvalues, Linear Multilinear Algebra 58(7-8)(2010)1053-1069.

[8] P. Lancaster, Theory of Matrices, Academic Press, New York and London, 1969.

[9] P.C. Müller, W.O. Schiehlen, Linear Vibrations, Martinus Nijhoff Publishers, Dordrecht Boston Lancaster, 1985.

[10] S.V. Savchenko, On the change in the spectral properties of a matrix under perturbations of sufficiently low rank, Funct. Anal. Appl. 38(1)(2004)69-71.

[11] J. Stoer, R. Bulirsch, Introduction to Numerical Analysis, Springer, New York Heidelberg, Third Edition, 2010.

[12] F. Stummel, K. Hainer, Introduction to Numerical Analysis, Scottish Academic Press, Edinburgh, 1980. 